\title{
Analysis of relationships between residual magnetic field and residual stress
}

\author{
Maciej Roskosz $\cdot$ Andrzej Rusin $•$ Michał Bieniek
}

Received: 14 November 2011 / Accepted: 31 July 2012 / Published online: 30 August 2012

(C) The Author(s) 2012. This article is published with open access at Springerlink.com

\begin{abstract}
The impact of stress on changes in magnetisation is one of the most complex issues of magnetism. Magnetic methods make use of the impact of stress on permeability, hysteresis and magnetic Barkhausen noise, which are examined with fields with a high strength and a small frequency. The paper presents an analysis of the impact of residual stress resulting from inhomogeneous plastic deformations in the notch area of the examined samples on the changes in the strength of the residual magnetic field (RMF). The RMF on the surface of the component is the superposition of the simultaneous effect of the shape, the anisotropic magnetic properties of the material, as well as of the values of the components of a weak external magnetic field (most commonly - the magnetic field of the Earth). Distributions of the RMF components were measured on the surface of samples with a various degree of plastic strain. The finite element method was used to model residual stress in the samples. The impact of residual stress on changes in the residual magnetic field was shown. A qualitative correlation was found between places with residual stress and areas with increased values of the gradients of the RMF components. Further research is now in progress in order to develop the quantitative relationships.
\end{abstract}

M. Roskosz $(\varangle) \cdot$ A. Rusin $\cdot$ M. Bieniek

The Silesian University of Technology, Konarskiego 18, 44-100 Gliwice, Poland

e-mail: maciej.roskosz@polsl.pl
Keywords Residual magnetic field · Residual stress · Plastic deformation

\section{Introduction}

Residual stress is the stress which occurs in construction components which are not subjected to external loads. Residual stresses of the first order can arise due to a gradient in plastic deformation caused either by mechanical deformation or by the thermal gradient during cooling [1-4].

The possibilities of evaluating applied stresses and residual stresses of the first order on the basis of the residual magnetic field (RMF) were indicated in [511]. [5-8] showed the possibility of the stress state evaluation on the basis of RMF measurements. In [6] the relationships between the gradient of the RMF normal component and stress measured with the $\mathrm{X}$ ray diffraction method were studied. In [5] the impact of the level and distribution of stress on the values of the RMF components was found for static tensile loads. For varying loads it was found that there was an influence of the stress amplitude and the number of cycles. L.H. Dong et al. [8] and C.L. Shi et al. [9] found a relationship between the gradient of the normal component and previously applied static tension load. S. Changliang et al. [10] report a considerable impact of the notch effect coefficient (the ratio of the max local stress at the notch under external load to the 
Table 1 Chemical composition of the sample material

\begin{tabular}{lllllll}
\hline Steel grade & \multicolumn{6}{l}{ Chemical composition of steel (\%) } \\
\cline { 2 - 7 } & $\mathrm{C}$ & $\mathrm{Si}$ & $\mathrm{Mn}$ & $\mathrm{P}$ & $\mathrm{S}$ & $\mathrm{N}$ \\
\hline $\mathrm{S} 235$ & $\max 0.17$ & - & $\max 1.40$ & $\max 0.045$ & $\max 0.045$ & $\max 0.009$ \\
\hline
\end{tabular}

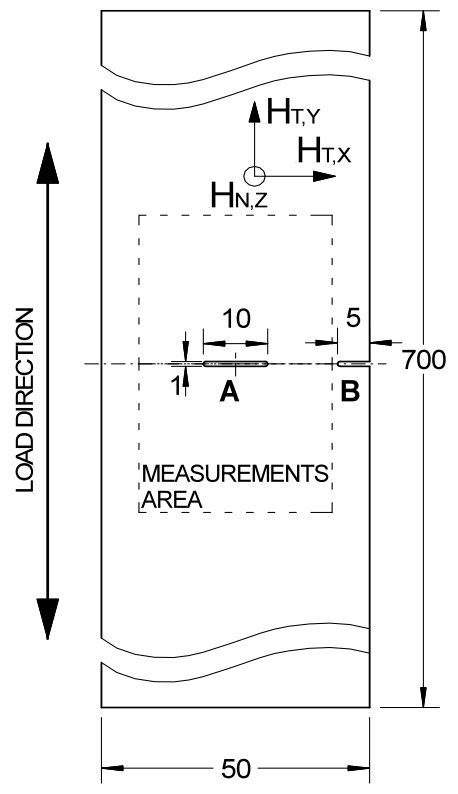

Fig. 1 Experimental samples (variant A-notch in the centre of the width of the sample; variant B-notch at the edge of the sample)

nominal stress without a notch) on the gradient of the normal component of the RMF.

This paper presents an analysis of qualitative relationships between the RMF distributions on the surface of notched samples with plastic deformations and the distributions of calculated values of residual stress. The presented results are a continuation of the research conducted with a view to determining the stress state based on magnetic parameters [3]. They extend the analysis of the relationships between residual stress of the first order and the RMF by a two-dimensional problem.

\section{Experimental details}

The samples were of the form of 2-mm thick flat bars made of S235 steel whose chemical composition is given in Table 1. A notch was made in the samples in

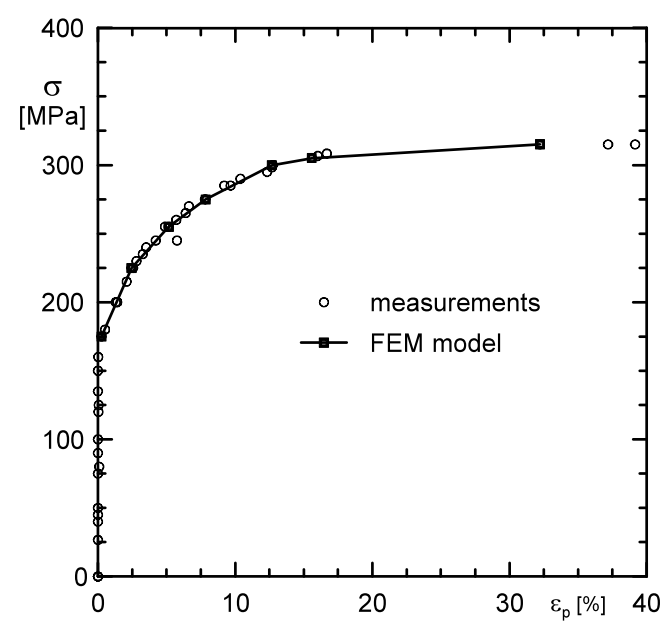

Fig. 2 Relationship between plastic strain and engineering stress for S235 steel

the shape of a slot in the centre of the sample - variant A in Fig. 1, and a slot on the edge of the samplevariant B in Fig. 1. The relationship between plastic strain and engineering stress for S235 steel is shown in Fig. 2.

The samples were loaded on a tensile testing machine Galdabini Sun 10P. After the desired loads were applied, the samples were unloaded and removed from the testing machine prior to being examined. The examination was always carried out at the same place and with the same position of the sample. 5 samples of each kind were analysed.

\subsection{Residual magnetic field measurements}

The magnetic field measurements were conducted in the "measuring area" marked in Fig. 1, with a scanning increment of $1 \mathrm{~mm}$ along vertical lines which were $4 \mathrm{~mm}$ apart from each other. The magnetometer TSC$1 \mathrm{M}-4$ with the measuring sensor TSC-2M supplied by Energodiagnostika Co. Ltd. Moscow was used for the measurements. The instrument was calibrated in the magnetic field of the Earth, whose value was assumed at $40 \mathrm{~A} / \mathrm{m}$. 


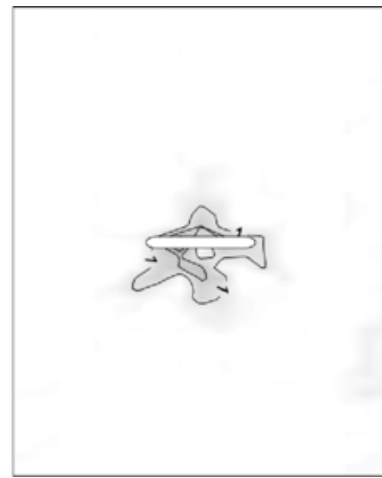

a) $\operatorname{grad} \mathrm{H}_{T, X}$,

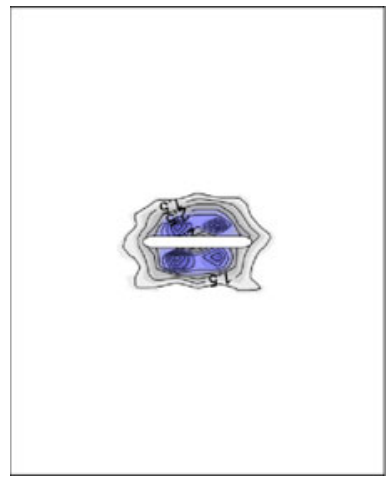

b) $\operatorname{grad} \mathrm{H}_{\mathrm{T}, \mathrm{Y}}$,

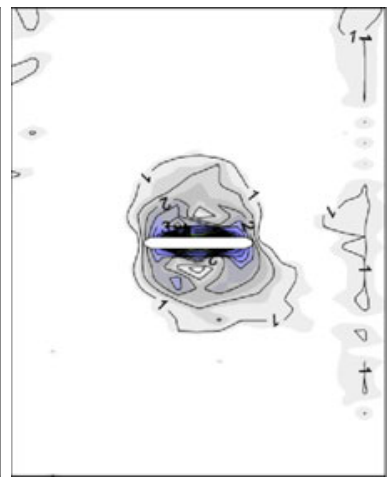

c) $\operatorname{grad} \mathrm{H}_{\mathrm{N}, \mathrm{Z}}$,

Fig. 3 Distributions of gradients of RMF components—initial state-variant A sample

The measurements resulted in the values of $3 \mathrm{RMF}$ components on the sample surface (Fig. 1):

- $H_{t, x}$-tangential component measured in the direction perpendicular to the applied load,

- $H_{t, y}$-tangential component measured in the direction parallel to the applied load,

- $H_{n, z}$-normal component.

The samples were not initially demagnetised; their degree of magnetisation varied slightly at the initial stage. The differences got smaller and smaller after the yield point of the material had been exceeded. The gradients of the RMF components are used to analyse the state of stress and deformation. If the initial distribution of the RMF on the surface of the sample is reasonably uniform and features a low level of RMF gradients, this indicates that there is no or very little residual stress of the first order after the manufacturing process. Example initial distributions of the gradients of the RMF components measured on the surface of the samples are shown in Figs. 3a to $3 \mathrm{c}$ for samples with a notch in the centre of the sample-variant A. The areas with increased values of RMF gradients which can be seen in the figures are related to the shape of the sample and the magnetic flux leakage in the vicinity of the notch.

\subsection{Residual stress calculations}

The residual stress values were determined by means of the finite element method (FEM). The software package Ansys 12.1 was used.

The tensile curve for steel S235 was approximated using a multilinear model Fig. 2. The material proper- ties were assumed as isotropic. Due to the small thickness of the samples, the problem was modelled as a two-dimensional one, assuming that it was a plane stress state. The numerical model mesh, which fully corresponded to the geometry of the samples, was built on the basis of eight-node quadrangular elements.

The boundary conditions included the fixing of the model on one hand, and the application of tensile loads corresponding to the force set by the strength testing machine on the other.

The way in which the calculations were carried out made it possible to take account of the plastic strain accumulation in each subsequent cycle of the loading of the sample, i.e. the stress-strain state determined in each calculation step constituted the initial state used to determine the stress-strain state in the next step. For the needs of the performed analyses, the results were derived for the area covered by the RMF measurements-Fig. 1.

\section{Results and discussion}

Due to the magnetoelastic effect, mechanical stress has an influence on the energy anisotropy of magnetic domains, which most often results in changes in permeability. The direction of the anisotropy depends on magnetostriction. For materials with positive magnetostriction, the magnetic moments tend to align in parallel to the direction of tensile stress, and perpendicular to compressive stress. In materials with negative magnetostriction, opposite phenomena occurthe magnetic moments tend to align perpendicular to the direction of tensile stress, and in parallel to compressive stress [12, 13]. 


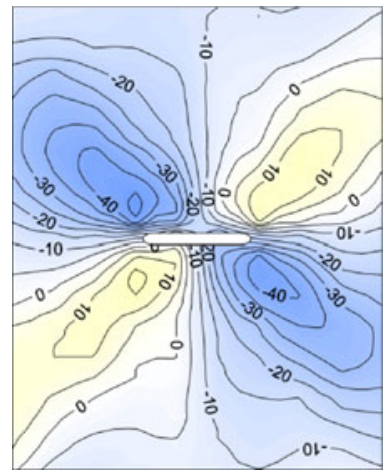

a) $\mathrm{H}_{T, \mathrm{X}}$,

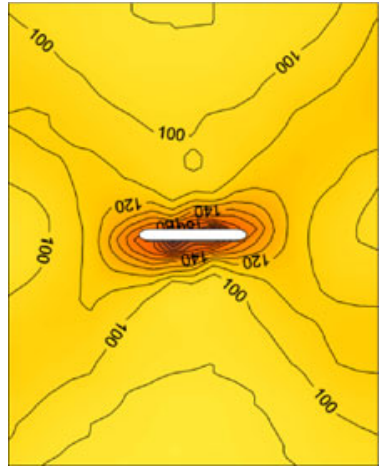

b) $\mathrm{H}_{\mathrm{T}, \mathrm{Y}}$



c) $\mathrm{H}_{\mathrm{N}, \mathrm{Z}}$

Fig. 4 Distributions of RMF components measured after the sample is unloaded (active tensile stress value $\sigma=225 \mathrm{MPa}$ )—variant A sample

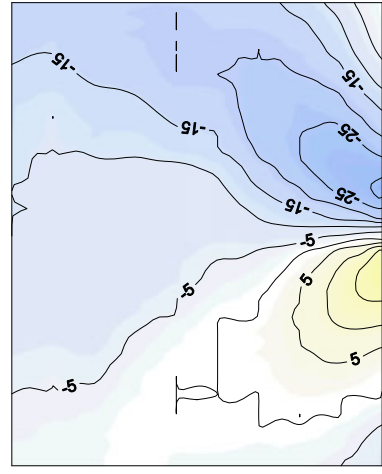

a) $\mathrm{H}_{T, X}$,



b) $\mathrm{H}_{\mathrm{T}, \mathrm{Y}}$

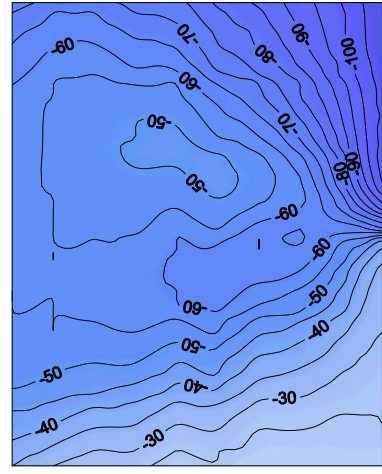

c) $\mathrm{H}_{\mathrm{N}, \mathrm{Z}}$

Fig. 5 Distributions of RMF components measured after the sample is unloaded (active tensile stress value $\sigma=275 \mathrm{MPa}$ ) — variant B sample

The impact of uniaxial stress on the domain structure can be compared to the effect of a magnetic field with strength $H_{\sigma}$ which is equivalent to the stress

$H_{\sigma}(\phi)=\frac{3}{2} \frac{\sigma}{\mu_{0}}\left(\frac{d \lambda}{d M}\right)_{T}\left(\cos ^{2} \phi-v \sin ^{2} \phi\right)$

where $\sigma$ is stress, $\lambda$-magnetostriction, $\mu_{0}$-magnetic permeability of free space, $M$-magnetisation, $\phi$ the angle between the stress axis and the direction of magnetic field $H_{\sigma}$, and $v$-Poisson's ratio [12-16]. Dependence (1) results from the fact that, in a certain range of the magnetic field strength $H$ and stress $\sigma$, Villary's effect is the inverse of Joule's effect and in this case these effects are interrelated by a thermodynamic dependence.

$\frac{1}{l}\left(\frac{d l}{d H}\right)_{\sigma}=\left(\frac{d M}{d \sigma}\right)_{H}$
In order to describe the impact of the complex stress state, the notion of equivalent stress is introduced, i.e. of a fictitious uniaxial stress whose amplitude will lead to the same change in susceptibility as real multiaxial stress [17-22]. The problems related to the impact of a complex stress state on changes in magnetisation are issues whose description and modelling, due to potential application for stress measurements, are the subject of current research [17-22].

The residual magnetic field of a ferromagnetic element, also known as the Self Magnetic Flux Leakage, is the sum of the simultaneous effect of the geometry of the object and of the magnetic, electrical and mechanical properties of the material of which it was made in the magnetic field of the Earth. Due to magnetomechanical coupling, the stress which occurs in the object (both active and residual) has an impact on 


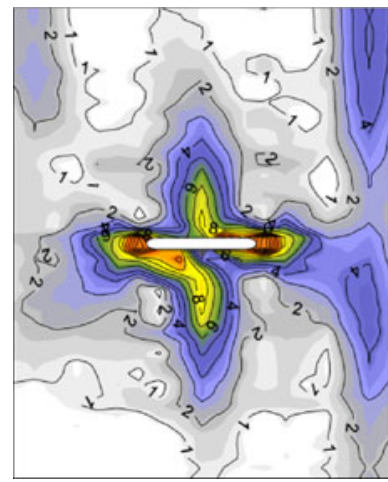

a) $\sigma=150 \mathrm{MPa}$, grad $\mathrm{H}_{\mathrm{T}, \mathrm{X}}$,

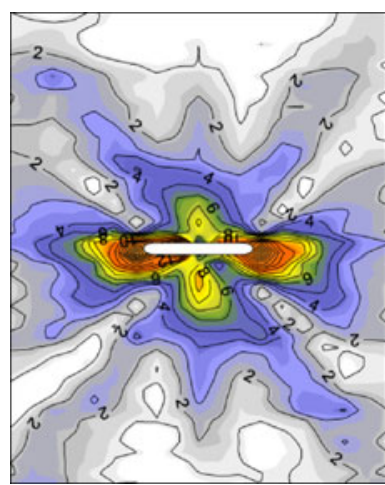

d) $\sigma=225 \mathrm{MPa}, \operatorname{grad} \mathrm{H}_{\mathrm{T}, \mathrm{X}}$,

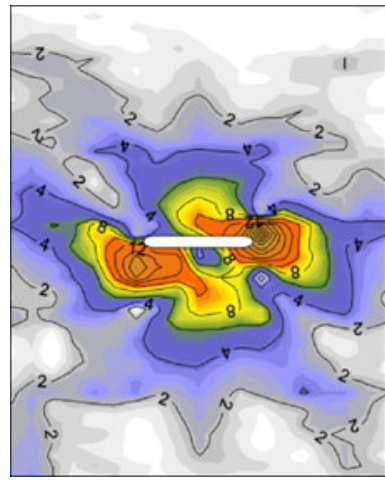

g) $\sigma=305 \mathrm{MPa}, \operatorname{grad} \mathrm{H}_{\mathrm{T}, \mathrm{X}}$,

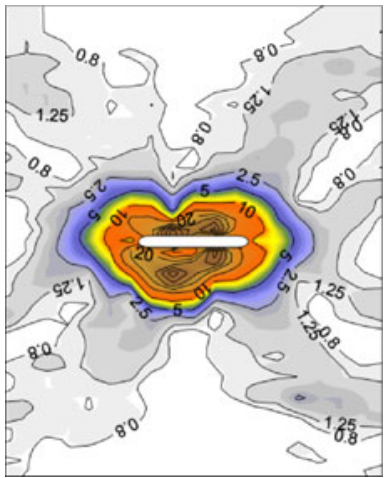

b) $\sigma=150 \mathrm{MPa}$, grad $\mathrm{H}_{\mathrm{T}, \mathrm{Y}}$,

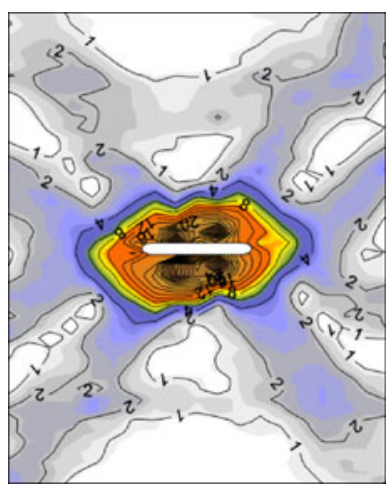

e) $\sigma=225 \mathrm{MPa}$, grad $\mathrm{H}_{\mathrm{T}, \mathrm{Y}}$,

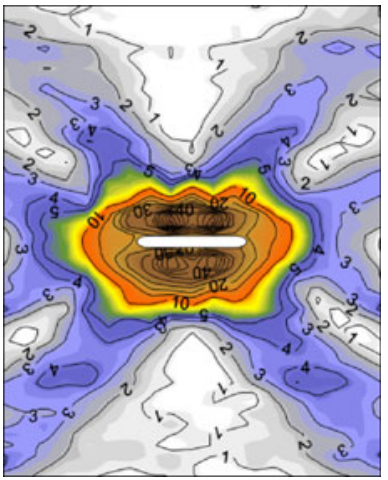

h) $\sigma=305 \mathrm{MPa}$, grad $\mathrm{H}_{\mathrm{T}, \mathrm{Y}}$,

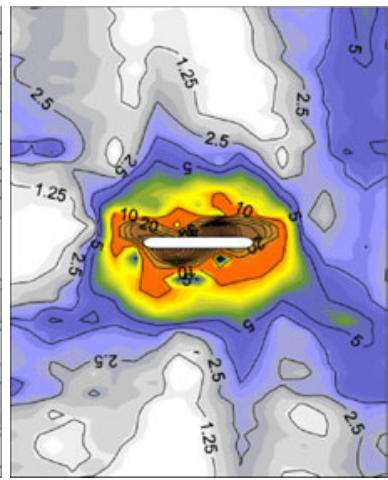

c) $\sigma=150 \mathrm{MPa}$, grad $\mathrm{H}_{\mathrm{N}, \mathrm{Z}}$,

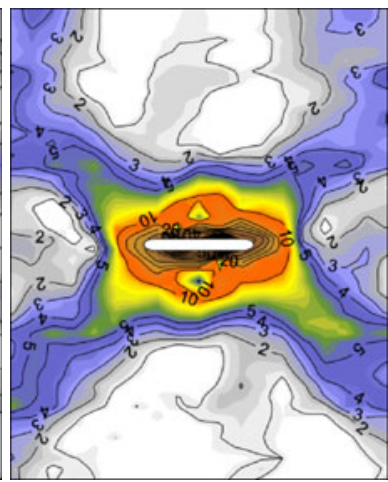

f) $\sigma=225 \mathrm{MPa}$, grad $\mathrm{H}_{\mathrm{N}, \mathrm{Z}}$,

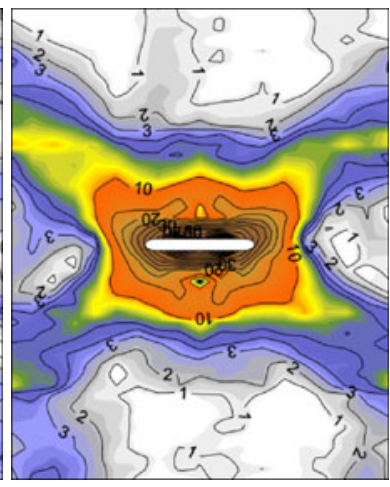

i) $\sigma=305 \mathrm{MPa}$, grad $\mathrm{H}_{\mathrm{N}, \mathrm{Z}}$,

Fig. 6 Distributions of gradients of RMF components measured after the sample is unloaded for different values of active tensile stress $\sigma$-variant A sample

the RMF. Assuming invariability of the object location in the magnetic field of the Earth, invariability of the Earth's magnetic field itself (the assumption is not fully true) and lack of significant changes in geometry (there are some slight variations caused by plastic strain), the changes in the RMF of the deformed object are caused by the action of stress resulting from magnetomechanical coupling (Villary's and Joule's effect).

In the presented analysis, and assuming positive magnetostriction, both the negative values of residual stress $\sigma_{X}$ and the positive values of residual stress $\sigma_{Y}$ should cause an increase in tangential component $H_{T, Y}$. 
Representative results of the RMF measurements in the "measuring area" marked in Fig. 1 of samples with plastic deformations are presented in this paper. The location and dimensions of the notch in variant $\mathrm{A}$ and B samples are presented in Fig. 1. For variant A samples, the measuring area includes the notch; for variant B samples - the notch is beyond the area. In the immediate vicinity of the notch, the distribution of the RMF gradients depends mainly on the magnetic flux leakage caused by the discontinuity of the material. In the remaining area, the RMF distribution is the result of the impact of a certain distribution of stress and of the properties of the material (such as local changes in structure, chemical composition) which affect magnetic properties. For the one-dimensional problem, the possibility of evaluating residual stress based on the gradients of the RMF components is presented in [3]. The presented results are a supplement and continuation of [3]. In further consideration, the concept of the gradient of the RMF component will be understood as its absolute value. Figures $6 \mathrm{a}$ to $6 \mathrm{i}$ illustrate the distributions of the gradients of the RMF components representative of variant A samples which are measured after unloading for different values of active tensile stress $\sigma$ understood as nominal stress in the cross-section weakened by the notch. Figures $8 \mathrm{a}$ to $8 \mathrm{i}$ are representative of variant $\mathrm{B}$ samples.

In the initial state, the effect of the magnetic flux leakage in the notch area dominates in the distributions of the gradients of the RMF components (example for a variant A sample in Figs. 3a to 3c). For a certain geometry, their values depend mainly on the outer magnetic field and on the magnetic permeability of the material. Due to the impact of the notch being a stress concentrator, even for slight values of active stress $\sigma$, small areas of plastic deformations appear in the area of the notch. A rise in active stress causes an increase in the size of the deformations. This results in changes in the values of the RMF components and of their gradients, which can be traced for a variant A sample for the gradient of the tangential component $H_{T, Y}$ from the initial state-Fig. $3 \mathrm{~b}$ through subsequent states for the nominal active stress value $\sigma=50 \mathrm{MPa}$-Fig. 7a, and $\sigma=100 \mathrm{MPa}$-Fig. 7b. A rise in the values of the gradients, as well as an increase and a slight change in the shape of the area where their increased values appear, can be noticed. A rise in the values of active stress leads to the formation of a characteristic distri-

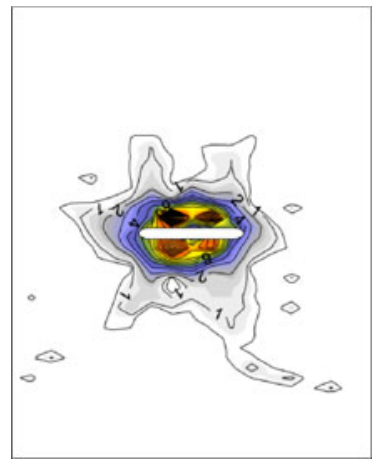

a) $\sigma=50 \mathrm{MPa}$,

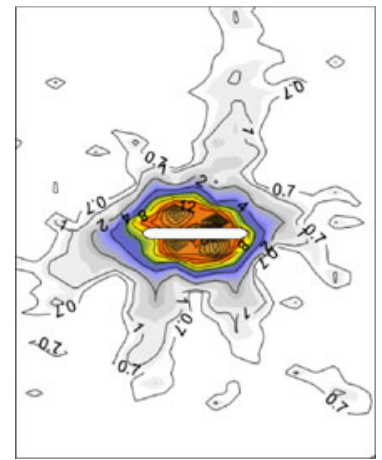

b) $\sigma=100 \mathrm{MPa}$,
Fig. 7 Changes in the gradient distribution of component $H_{T, Y}$ for slight nominal stress values $\sigma$ in the cross-section weakened by the notch-variant A sample

bution of the RMF components after the sample is unloaded. An example of such a distribution for a variant A sample with a notch in the centre is shown in Figs. $4 \mathrm{a}$ to $4 \mathrm{c}$. For a variant B sample with a notch at the edge, the distributions are presented in Figs. 5a to $5 c$. Further changes in stress values cause changes in the values of the gradients of the RMF components, but the configuration of isolines remains practically the same. The impact of the increase in the values of active stress $\sigma$ (and of residual stress, respectively) on the gradient values and distributions can be analysed in Figs. 6a to 6i (variant A sample) and 8a to 8i (variant B sample). At a certain level of the nominal active stress value (for the samples under consideration it is about $150 \mathrm{MPa}$ ), due to the notch effect, the yield point is exceeded locally. Inhomogeneous deformations and residual stress of the first order appear. Characteristic gradient distributions (resulting from the geometry of the samples and from its impact on the stress distribution) are formed. The values of the gradients of the RMF components rise together with the increase in the values of residual stress.

The results of the FEM modelling of the residual stress components for a variant A sample are presented in Figs. 9a to 9f-components $\sigma_{x}$ and $\sigma_{y}$, and in Fig. 11a-component $\tau_{x y}$. Similarly, for a variant B sample, Figs. 10a to 10f present components $\sigma_{x}$ and $\sigma_{y}$ and Fig. $11 \mathrm{~b}$ shows component $\tau_{x y}$. Figures $9 \mathrm{a}$ to 9f and 10a to 10f illustrate the distributions of residual stress values for different levels of active tensile stress $\sigma$ ( $\sigma$-nominal stress in the cross-section weakened by the notch). 


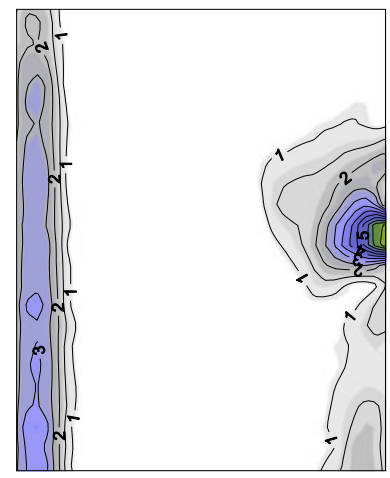

a) $\sigma=150 \mathrm{MPa}, \operatorname{grad} \mathrm{H}_{\mathrm{T}, \mathrm{X}}$,

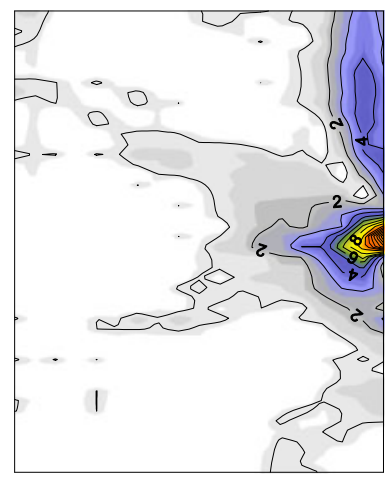

d) $\sigma=205 \mathrm{MPa}, \operatorname{grad} \mathrm{H}_{\mathrm{T}, \mathrm{X}}$,

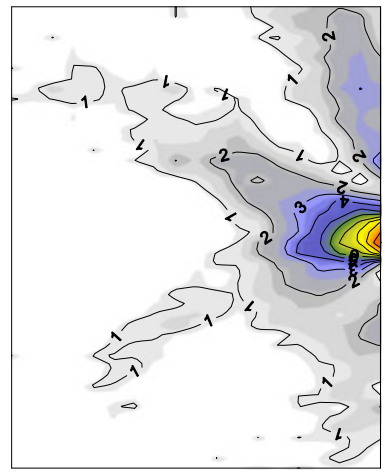

g) $\sigma=275 \mathrm{MPa}, \operatorname{grad} \mathrm{H}_{\mathrm{T}, \mathrm{X}}$,

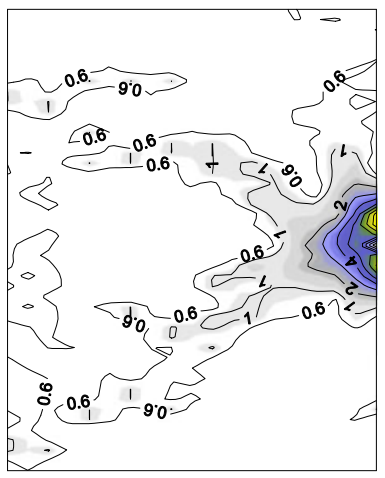

b) $\sigma=150 \mathrm{MPa}$, grad $\mathrm{H}_{\mathrm{T}, \mathrm{Y}}$,

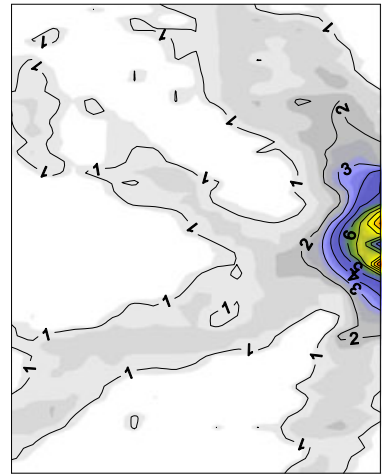

e) $\sigma=205 \mathrm{MPa}, \operatorname{grad} \mathrm{H}_{\mathrm{T}, \mathrm{Y}}$,

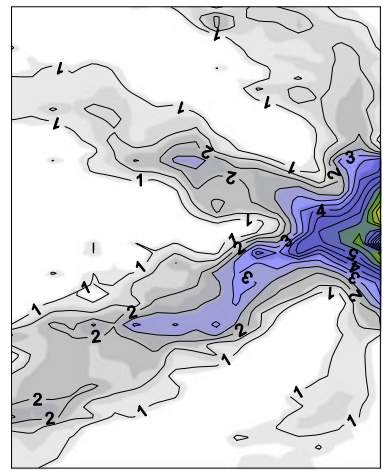

h) $\sigma=275 \mathrm{MPa}, \operatorname{grad} \mathrm{H}_{\mathrm{T}, \mathrm{Y}}$,

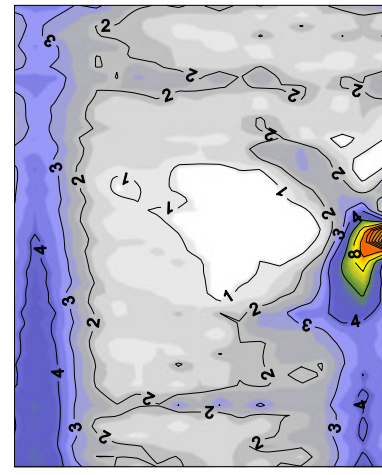

c) $\sigma=150 \mathrm{MPa}, \operatorname{grad} \mathrm{H}_{\mathrm{N}, \mathrm{Z}}$,

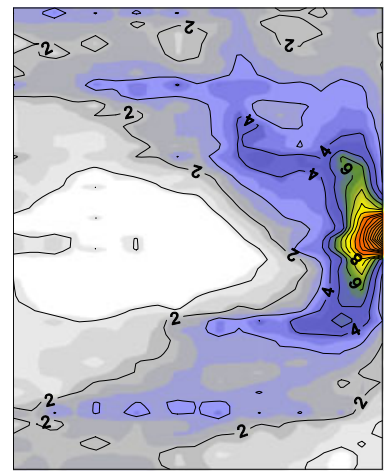

f) $\sigma=205 \mathrm{MPa}, \operatorname{grad} \mathrm{H}_{\mathrm{N}, \mathrm{Z}}$,

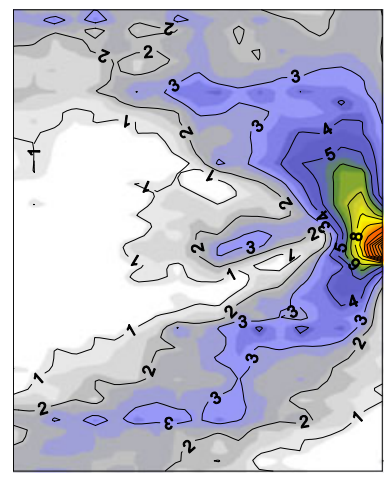

i) $\sigma=275 \mathrm{MPa}, \operatorname{grad} \mathrm{H}_{\mathrm{N}, \mathrm{Z}}$,

Fig. 8 Distributions of gradients of RMF components measured after unloading for different values of active tensile stress $\sigma$-variant B sample

A significant similarity is found between the distributions of the RMF gradients and the distributions of residual stress values. For a variant A sample, the distributions of residual stress $\sigma_{x}$ (Figs. 9a, 9c, 9e) resemble a flattened $X$, and the residual stress distributions $\sigma_{y}$ (Figs. 9b, 9d, 9f) look like an elongated $X$ (the centres of $X$ are located on the centre of the notch). In the image of the gradients (especially those of the tangen- tial components $H_{T, Y}$-Figs. 6b, 6e, 6h), shapes similar to two $X$ 's can be distinguished, which shows the impact of both $\sigma_{x}$ and $\sigma_{y}$ residual stress values. For a variant B sample, the distributions of residual stress $\sigma_{x}$ (Figs. 10a, 10c, 10e) and residual stress $\sigma_{y}$ (Figs. 9b, 9d, 9f) resemble rotated $V$ 's, whose arms come out of the notch. In the image of the gradients, similarly to the sample with the notch in the centre, for gradients of 


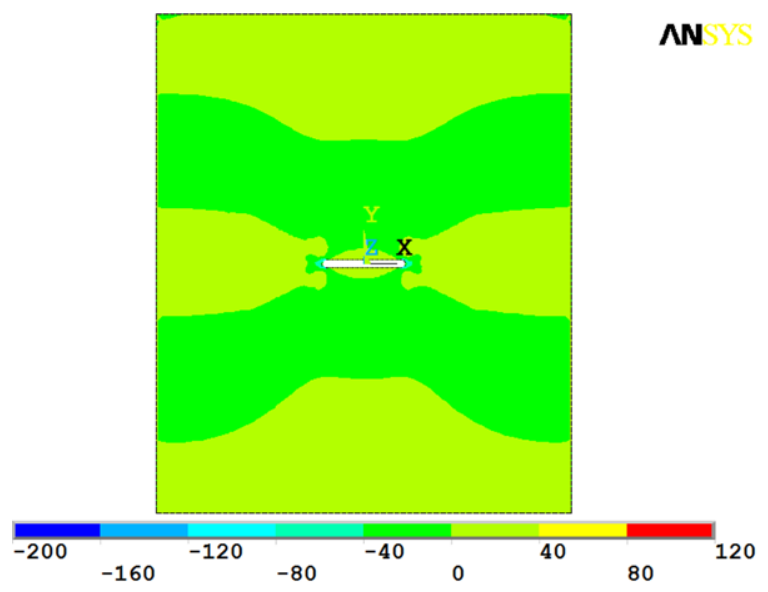

a) $\sigma=150 \mathrm{MPa}, \sigma_{\mathrm{x}}$

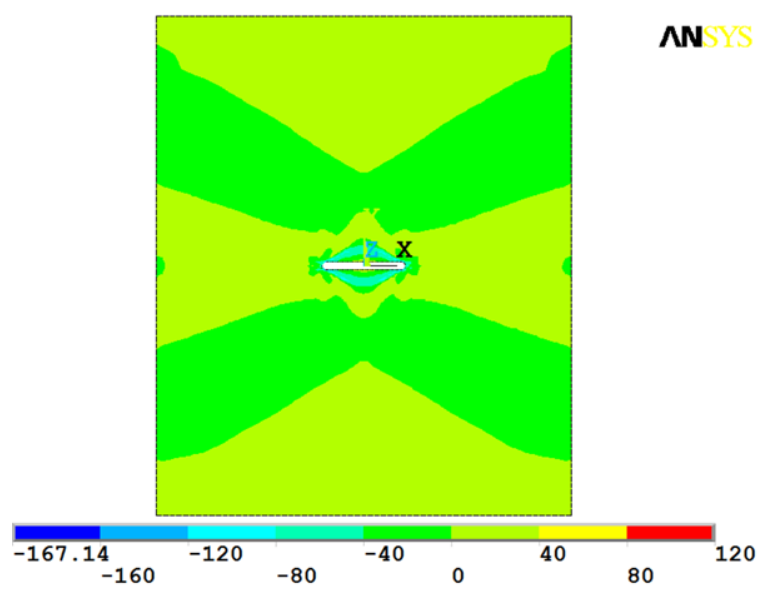

c) $\sigma=225 \mathrm{MPa}, \sigma_{\mathrm{x}}$

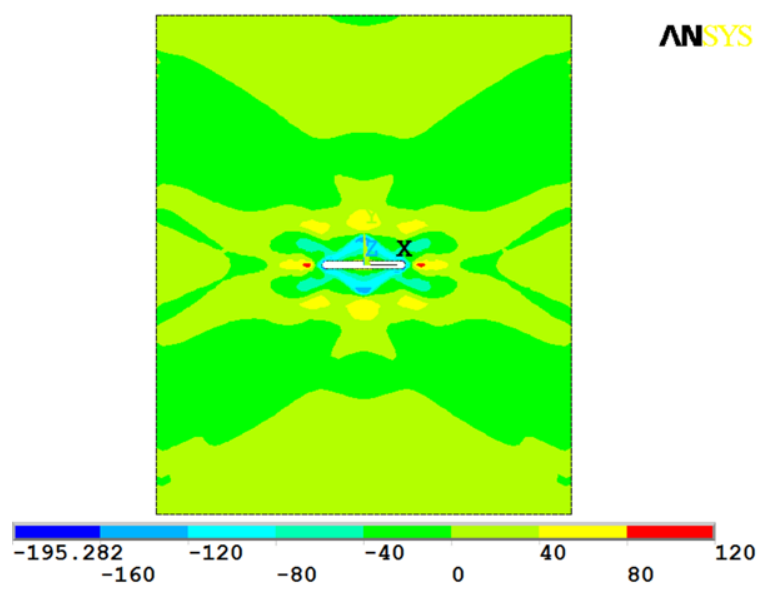

e) $\sigma=305 \mathrm{MPa}, \sigma_{\mathrm{x}}$

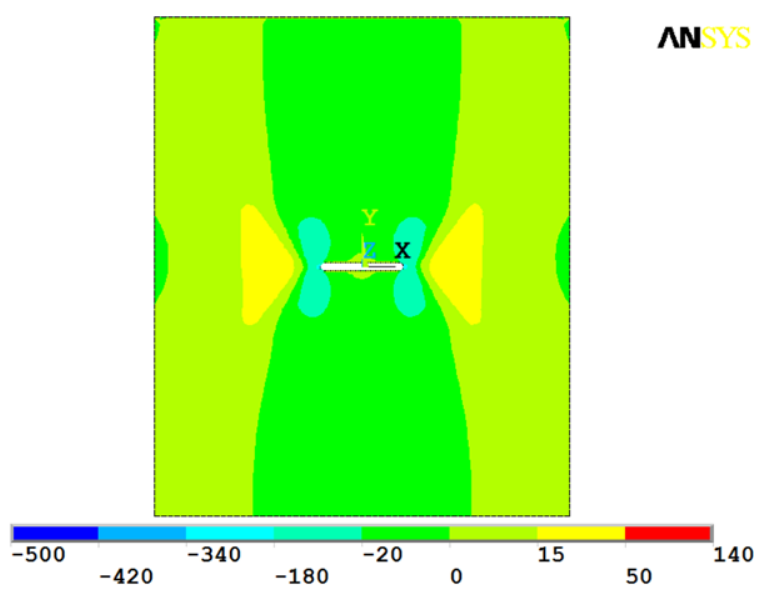

b) $\sigma=150 \mathrm{MPa}, \sigma_{\mathrm{y}}$

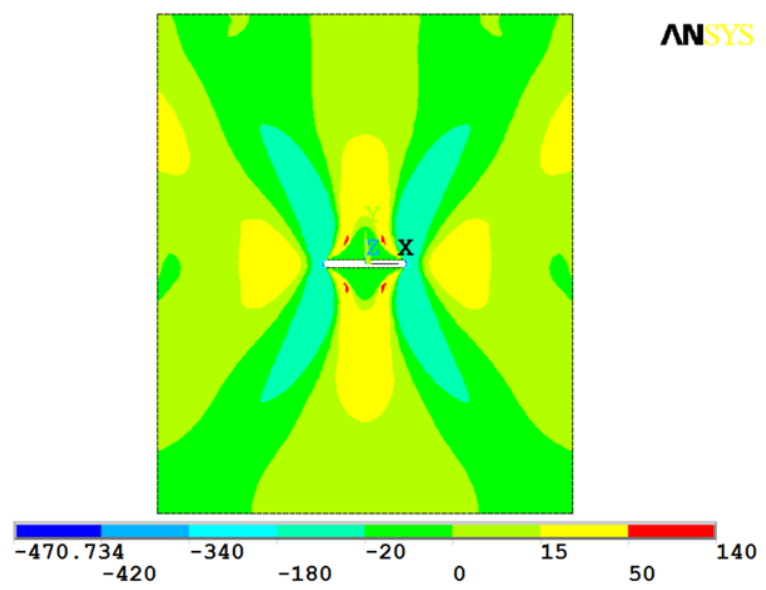

d) $\sigma=225 \mathrm{MPa}, \sigma_{\mathrm{y}}$

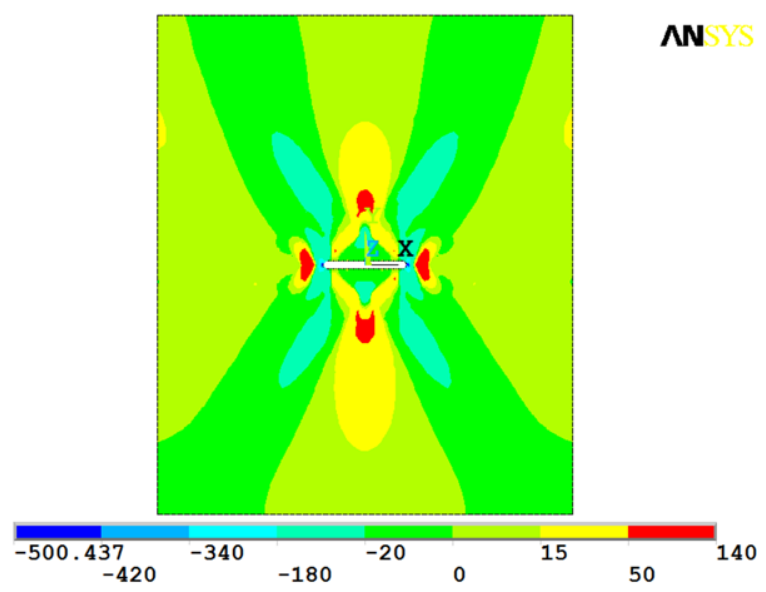

f) $\sigma=305 \mathrm{MPa}, \sigma_{\mathrm{y}}$

Fig. 9 Distributions of residual stress after the impact of different values of active tensile stress $\sigma$-variant A sample 


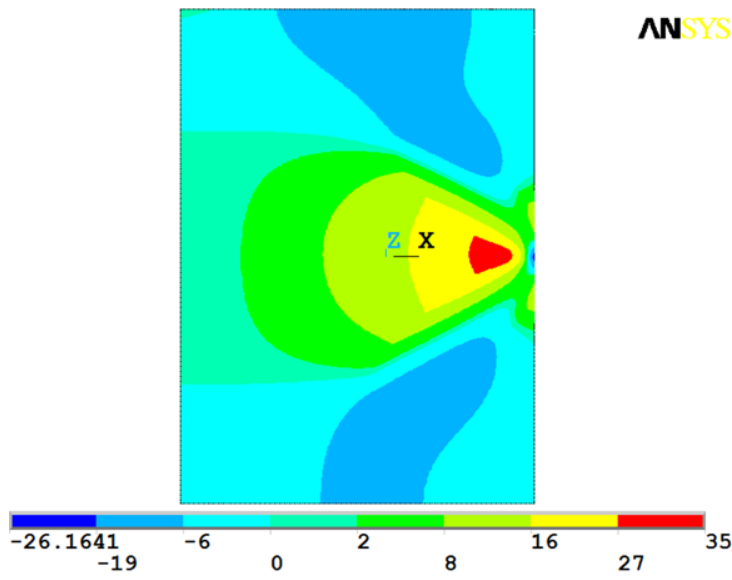

a) $\sigma=150 \mathrm{MPa}, \sigma_{\mathrm{x}}$

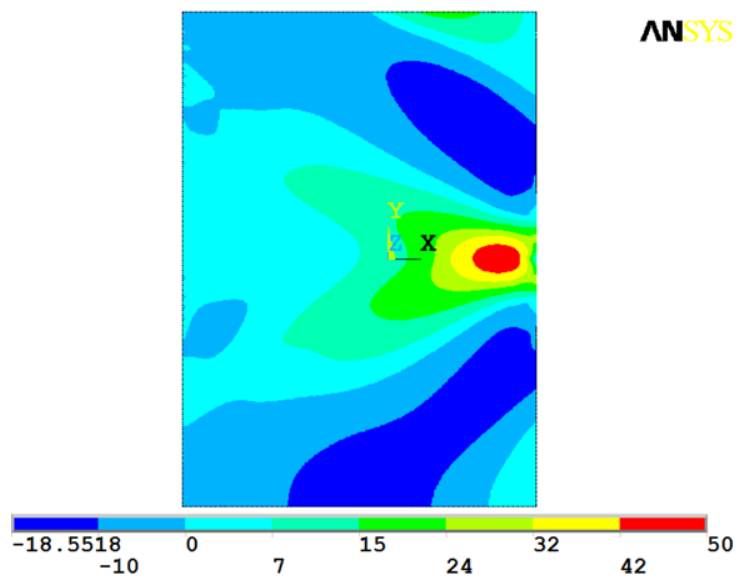

c) $\sigma=205 \mathrm{MPa}, \sigma_{\mathrm{x}}$

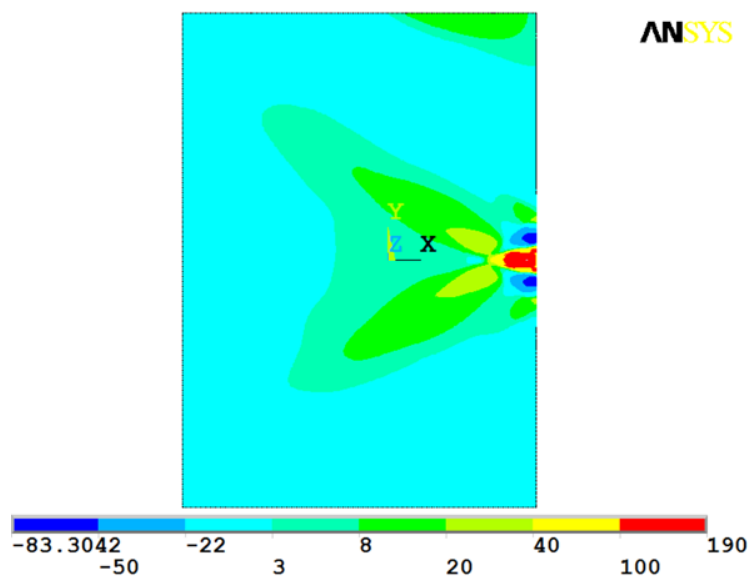

e) $\sigma=305 \mathrm{MPa}, \sigma_{\mathrm{x}}$

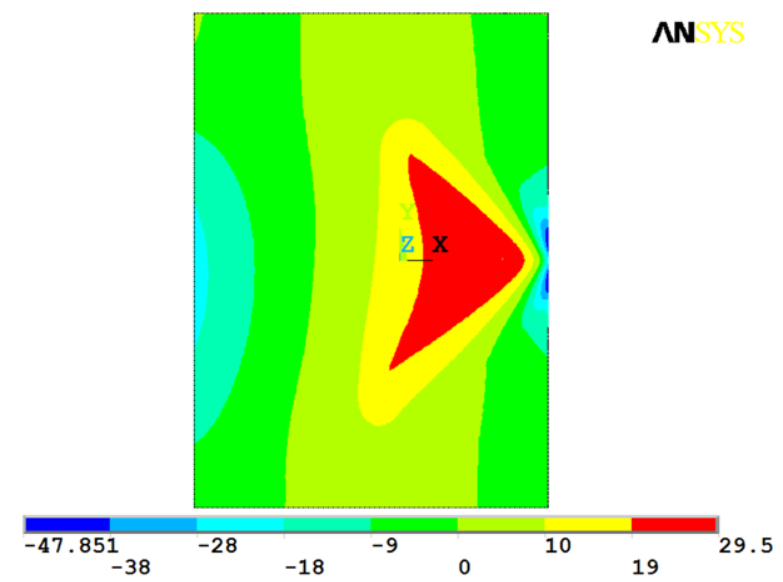

b) $\sigma=150 \mathrm{MPa}, \sigma_{\mathrm{y}}$

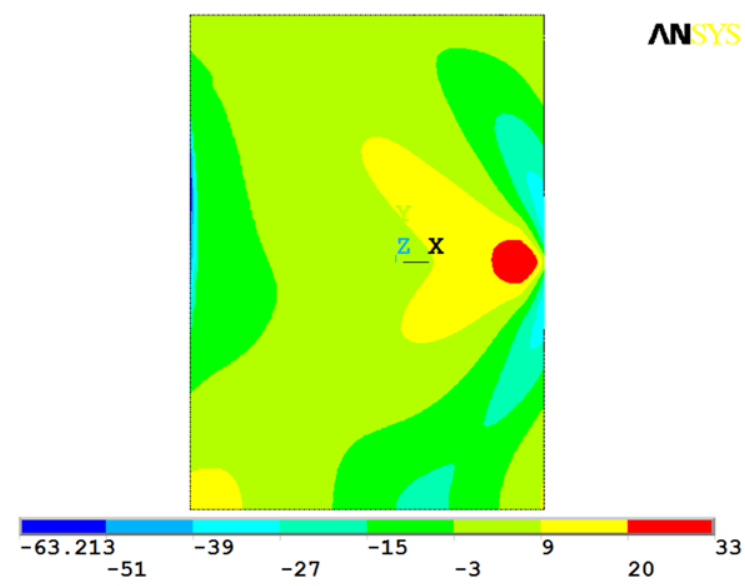

d) $\sigma=205 \mathrm{MPa}, \sigma_{\mathrm{y}}$

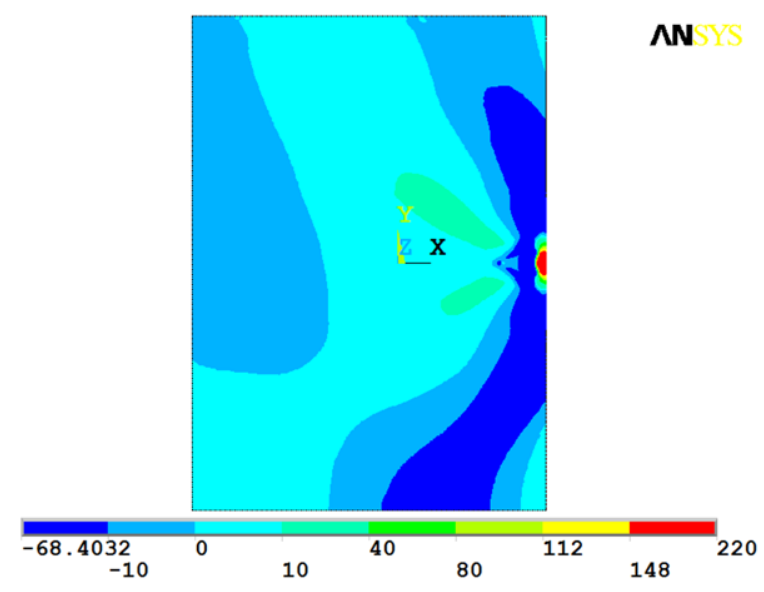

f) $\sigma=305 \mathrm{MPa}, \sigma_{\mathrm{y}}$

Fig. 10 Distributions of residual stress components for different values of active tensile stress $\sigma$ - variant B sample 


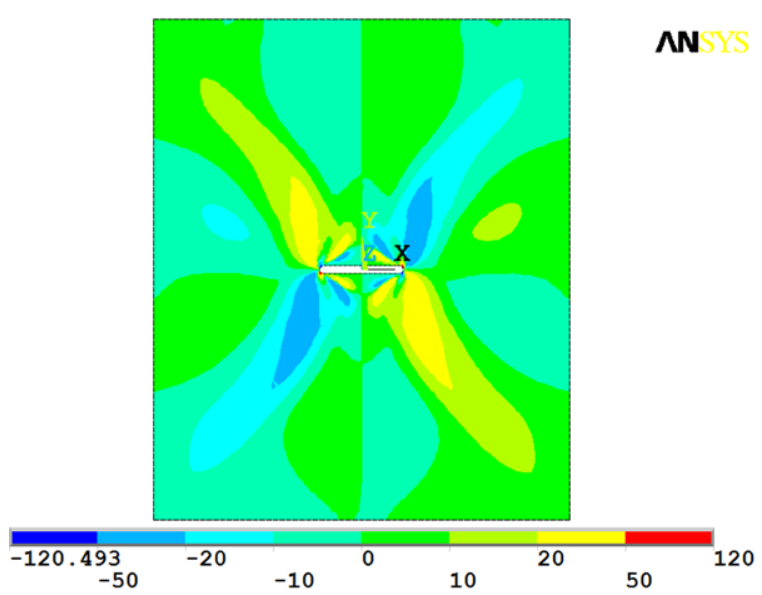

a) $\sigma=275 \mathrm{MPa}, \tau_{\mathrm{xy}}-$ variant A sample



b) $\sigma=225 \mathrm{MPa}, \tau_{\mathrm{xy}}-$ variant B sample

Fig. 11 Example distributions of shear residual stress $\tau_{x y}$ after the impact of different values of active tensile stress $\sigma$ for two variants of analysed samples

the tangential components $H_{T, Y}$-Figs. $8 \mathrm{~b}, 8 \mathrm{e}, 8 \mathrm{~h}-$ shapes that look like two $V$ 's can be distinguished. Higher gradient values correspond to higher residual stress values.

Generally, it can be stated that there are qualitative relationships between plastic deformations and the RMF magnitude, as well as between the RMF gradients and residual stress.

\section{Conclusions}

It is shown that for the samples under analysis the distributions of residual stress components are reflected in the distributions of the RMF gradients. This is particularly visible for the RMF tangential component.

There are qualitative relationships between RMF gradients and residual stress of the first order. This provides a basis for further research aiming at the development of quantitative relationships, whose concept for one-dimensional problems is presented in [3] and [23]. The research will make it possible to evaluate the state of the material by means of the RMF measurements for two-dimensional problems.

Open Access This article is distributed under the terms of the Creative Commons Attribution License which permits any use, distribution, and reproduction in any medium, provided the original author(s) and the source are credited.

\section{References}

1. Altpeter I, Dobmann G, Kröning M, Rabung M, Szielasko $S$ (2009) Micro-magnetic evaluation of micro residual stresses of the IInd and IIIrd order. NDT E Int 42:283-290

2. Withers PJ, Turski M, Edwards L, Bouchard PJ, Buttle DJ (2008) Recent advances in residual stress measurement. Int J Press Vessels Piping 85:118-127

3. Roskosz M, Bieniek M (2012) Evaluation of residual stress in ferromagnetic steels based on residual magnetic field measurements. NDT E Int 45:55-62

4. McEvily AJ (2002) Metal failures: mechanisms, analysis, prevention. Willey, New York

5. Roskosz M, Gawrilenko P (2008) Analysis of changes in residual magnetic field in loaded notched samples. NDT E Int 41:570-576

6. Kuroda M, Yamanaka S, Yamada K, Isobe Y (2001) Evaluation of residual stresses and plastic deformations for ironbased materials by leakage magnetic flux sensors. J Alloys Compd 314:232-239

7. Wilson JW, Tian GY, Barrans S (2007) Residual magnetic field sensing for stress measurement. Sens Actuators A $135: 381-387$

8. Dong L, Xu B, Dong S, Song L, Chen Q, Wang D (2009) Stress dependence of the spontaneous stray field signals of ferromagnetic steel. NDT E Int 42:323-327

9. Shi CL, Dong SY, Xu BS, He P (2010) Metal magnetic memory effect caused by static tension load in a casehardened steel. J Magn Magn Mater 322:413-416

10. Shi C, Dong S, Xu B, He P (2010) Stress concentration degree affects spontaneous magnetic signals of ferromagnetic steel under dynamic tension load. NDT E Int 43:8-12

11. Li J, Xu M (2012) Influence of uniaxial plastic deformation on surface magnetic field in steel. Meccanica 47:135-139. doi:10.1007/s11012-011-9424-2

12. Mierczak LL, Jiles DC, Fantoni G (2011) A new method for evaluation of mechanical stress using the reciprocal am- 
plitude of magnetic Barkhausen noise. IEEE Trans Magn 47(2):459-465.

13. Yamasaki T, Yamamoto S, Hirao M (1996) Effect of applied stresses on magnetostriction of low carbon steel. NDT E Int 29(5):263-268

14. Kaminski DA, Jiles DC, Biner SB, Sablik MJ (1992) Angular dependence of the magnetic properties of polycrystalline iron under the action of uniaxial stress. J Magn Magn Mater 104-107:382-384

15. Li L, Jiles DC (2003) Modified law of approach for the magnetomechanical model: application of the Rayleigh law to stress. IEEE Trans Magn 39(5):3037

16. Jiles DC (1995) Theory of the magnetomechanical effect. J Phys D, Appl Phys 28:1537

17. Kashiwaya K (1991) Fundamentals of nondestructive measurement of biaxial stress in steel utilizing magnetoelastic effect under low magnetic field. Jpn J Appl Phys 30:2932

18. Schneider CS, Richardson JM (1982) Biaxial magnetoelasticity in steels. J Appl Phys 53:8136
19. Sablik MJ, Riley LA, Burkhardt GL, Kwun H, Cannell PY, Watts KT, Langman RA (1994) Micromagnetic model for biaxial stress effects on magnetic properties. J Magn Magn Mater 132:131-148

20. Pearson J, Squire PT, Maylin MG, Gore JG (2000) Biaxial stress effects on the magnetic properties of pure iron. IEEE Trans Magn 36(5):3251-3253

21. Daniel L, Hubert O (2010) Equivalent stress criteria for the effect of stress on magnetic behavior. IEEE Trans Magn 46(8):3089-3092

22. Bulte DP (2009) The pursuit of hysteresis in polycrystalline ferromagnetic materials under stress. IEEE Trans Magn 45(1):83

23. Roskosz M, Bieniek M (2011) Analysis of the similarity between residual magnetic field distribution and the stressstrain state for 7CrMoVTiB10-10 (T/P24) steel. In: Rubinacci G, Tamburrino A, Villone F, Takagi T (eds) JSAEM studies in applied electromagnetics and mechanics, Applied Electromagnetic and Mechanics, vol 14, pp 221-222 